\title{
Sensory Processing Dysfunction in Children with Autism Spectrum Disorder
}

\author{
Rabindran $^{1 *}$, Darshini Madanagopal ${ }^{2}$, Shasidaran $^{3}$
}

${ }^{1}$ Consultant, Neonatologist, Billroth Hospital, Chennai, India

${ }^{2}$ Assistant Professor, Department of Psychology, Institute of Distance Education, University of Madras, Chennai, India

${ }^{3}$ Senior Resident, Department of Radiology, S.R.M. Medical College and Research Centre, Chennai, India

Autistic Spectrum Disorder (ASD) is characterised by difficulties in social communication and interaction. As per latest DSM 5 update variations in sensory responses were included in diagnostic spectrum of ASD. Hyper/hypo reactivity to sensory input explains the abnormal patterns of behaviours, activities or interests seen in children with ASD. Autistic children have a dysfunctional sensory system known as Sensory Integration Disorder, Sensory Integration Dysfunction or Sensory Processing Disorder (SPD). In SPD, there is difficulty in processing information from the 5 classic senses, the sense of movement, and/or the positional sense. There are 2 classification systems for SPD. According to Miller's Classification, there are three kinds of SPD namely Sensory Modulation Disorder (SMD), Sensory Based Motor Disorder and Sensory Discrimination Disorder. There are three subtypes in SMD namely OverResponsiveness, Under-Responsiveness and Sensory -Craving. According to Schaaf's Classification, there are five kinds of SPD namely Poor Sensory Perception, Somato Dyspraxia, Visuo Dyspraxia, Vestibular \& Bilateral Integration and Sensory Reactivity. Under Sensory Reactivity there is Hyperactivity and Hypoactivity. The symptomatology of sensory dysfunction varies based on the primary senses which are predominantly involved. They may present with tactile, vestibular, proprioceptive, visual input, auditory input, taste input or smell input dysfunction. There are plenty of assessment tools for SPD diagnosis. The prominent tools include Sensory Profile, Sensory Integration and Praxis Test, Sensory Processing Measure and Evaluation in Ayres Sensory Integration. Awareness regarding the SPD in children with ASD will help to understand the disorder better ultimately leading to proper management.

Keywords: Sensory Processing Dysfunction, Autism Spectrum Disorder, Short Sensory Profile.

Copyright @ 2020: This is an open-access article distributed under the terms of the Creative Commons Attribution license which permits unrestricted use, distribution, and reproduction in any medium for non-commercial use (NonCommercial, or CC-BY-NC) provided the original author and source are credited.

\section{AUTISTIC SPECTRUM DISORDER}

Autistic Spectrum Disorder (ASD) is characterised by difficulties in social communication and interaction. ASD is characterised by presence of restricted and repetitive patterns of behaviours, activities or interests [1]. According per the latest DSM 5 update variations in sensory responses were included in diagnostic spectrum of ASD. Since the update in 2013 of DMS-5, Sensory Processing Dysfunction in autistic children are being studied extensively. As per DSM-5, Hyper or hypo reactivity to sensory input or uncommon interests in sensory aspects of the environment may explain the abnormal patterns of behaviours, activities or interests seen in children with ASD. Briefly a child with ASD processes sensory information in the environment differently from other normal children.

\section{Sensory Processing Disorder (SPD) / Sensory Integration Disorder (SID)}

Sensory integration is an innate neurobiological process. Sensory Integration is the method of integration and interpretation of sensory stimulation from the environment by the brain. Autistic children have a dysfunctional sensory system. This condition is referred to as Sensory Integration Disorder (SID) [2]. Sensory integration disorder is synonymously known as Sensory Integration Dysfunction or Sensory Processing Disorder. In this neurological condition, there is difficulty in processing information from the 5 classic senses (vision, auditory, touch, olfaction, and taste), the sense of movement (vestibular system), and / or the positional sense (proprioception). In children with SID, sensory information is detected usually, however perceived abnormally and also the information is analyzed by the brain differently which ends up in distress or confusion. In this condition, sensory input is 
not integrated appropriately in the brain. This abnormality in integration leads to abnormal interpretation of environmental sensory stimuli. This ultimately leads to problems in development, information processing, and behaviour. Usually the sensory receptors are situated in the peripheral nervous system. However the sensory problems seen in ASD seem to arise from the neurological dysfunction within the central nervous system [3]. One or more senses may be either over- or under-reactive to environmental stimulation. This abnormal reaction to stimulation may lead to abnormal behaviours like hand-flapping, spinning and rocking.

\section{Classification of SPD}

There are 2 classification systems for SPD. One is Miller's Classification and the other is Schaaf's classification. According to Miller's Classification, there are three kinds of SPD namely Sensory Modulation Disorder, Sensory Based Motor Disorder and Sensory Discrimination Disorder [3].

Miller's Classification of Sensory Processing Disorder

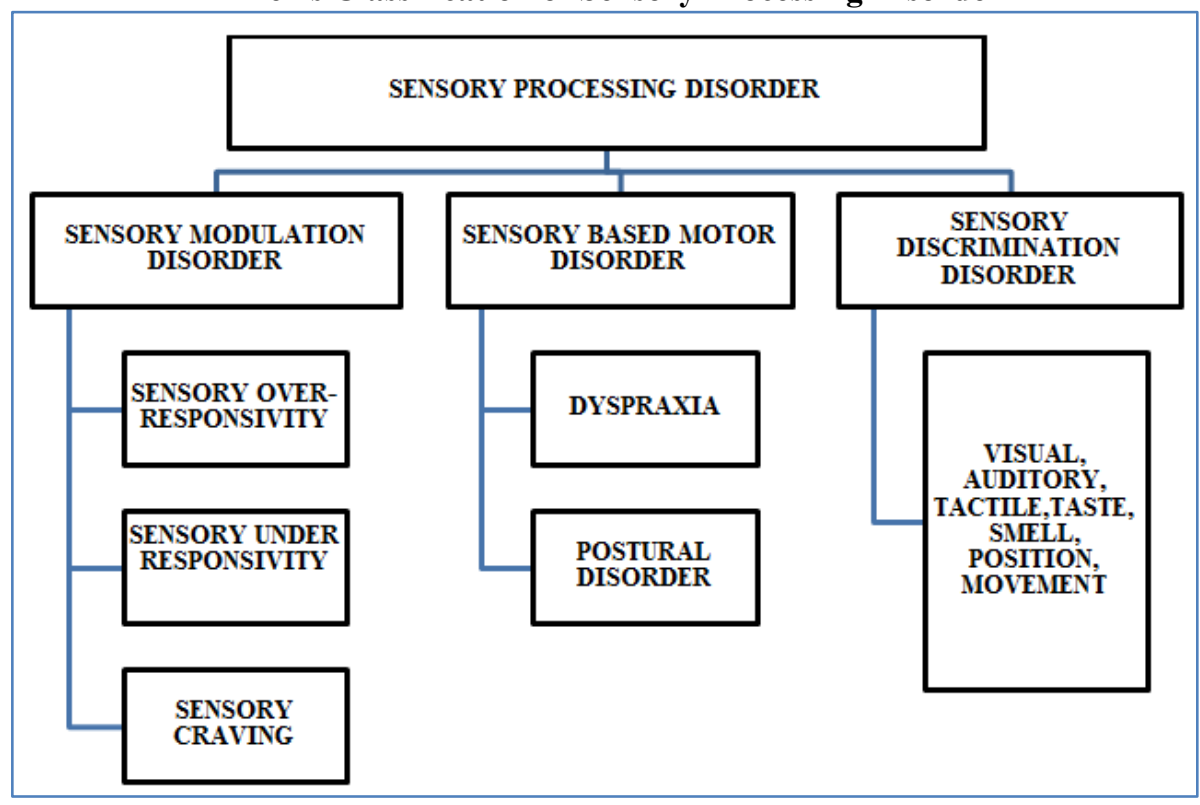

\section{Type I- Sensory Modulation Disorder (SMD)}

Sensory modulation is an advanced central nervous system process. Through modulation neural messages from the environment that convey information about the intensity, frequency, duration, complexity, and novelty of sensory stimuli are adjusted. The pathology in this sensory modulation leads to SMD in which there is over or under responding to sensory stimuli. Examples of SMD include a fearful and / or anxious pattern, negative and/or stubborn behaviours and self-involved behaviour.

There are three subtypes in SMD namely Over-Responsiveness, Under-Responsiveness and Sensory - Craving

\section{Over-Responsiveness}

Children with Over-Responsiveness have a tendency to perceive environmental input much stronger than a normal tendency. Hence they struggle to avoid many sensory experiences they may present with Tactile Defensiveness - difficulty to bear with any kind of touch or Gravitational Insecurity - a tendency to react negatively and fearfully to movement experiences, particularly those involving a change in head position and movement backward or upward through space.

\section{Under-Responsiveness}

Children with Under-Responsiveness have a tendency to avoid many sensory experiences as they register the environmental input less than a normal amount. Thy have Sensory Registration Problemsmanifested by failure to notice stimuli that normally are salient to most people. They may need larger sensory input to stimulate themselves as compared to other typical child [4].

\section{Sensory-Craving}

Unlike the previous two categories, children in this type crave for any kind of sensory input. These children present with excessively touching objects, Extreme body movements and eating solid food and changing quickly from one food item to other. Because of their sensory craving these children might try several activities.

\section{Type II- Sensory Based Motor Disorder (SBMD)}

Sensory Based Motor Disorder is a type of SPD in which there is disorganisation of motor output due to incorrect processing of sensory information. 
Type III- Sensory Discrimination Disorder (SDD)

Sensory discrimination disorder includes postural control challenges and / or dyspraxia. Examples of SDD include inattentiveness, disorganization and poor school performance.
According to Schaaf's Classification, there are five kinds of SPD namely Poor Sensory Perception, SomatoDyspraxia, VisuoDyspraxia, Vestibular \& Bilateral Integration and Sensory Reactivity. Under Sensory Reactivity there is Hyperactivity and Hypoactivity [5].

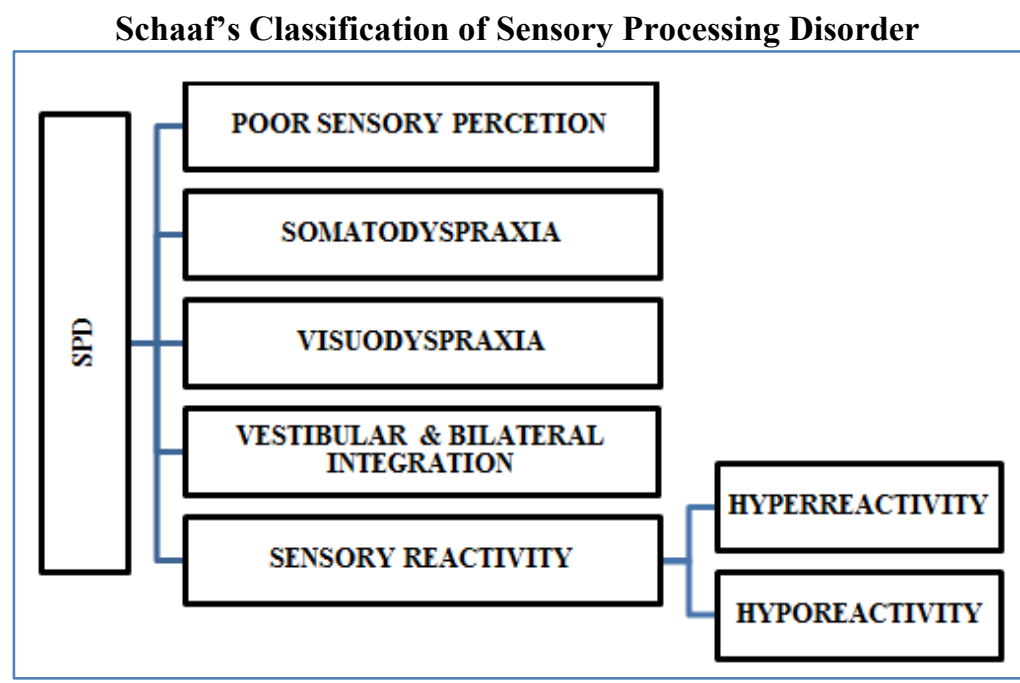

\section{Implications of Sensory Processing Disorder}

Sensory Dysfunction manifests itself in many ways. A child may be over or under-responsive to sensory input; activity level may be high or low; a child may like constant motion or fatigue easily [6]. Sometimes a child may have several attributes of sensory dysfunction and may fluctuate between these extremes. Due to this there may be Gross and / or fine motor coordination problems. They may have speech / language delays and thereby land in educational underachievement [7]. There could also be associated Behavioural disorders and therefore the child may be impulsive, distractible, and have a general lack of planning. Typically this might cause problem in adjusting to new situations thereby resulting in frustration, aggression, or withdrawal.

\section{Clinical Presentation of Sensory Processing Dysfunction in ASD Children}

The symptomatology of sensory dysfunction varies based on the primary senses which are predominantly involved.

\section{Tactile Dysfunction}

Skin is the largest organ of the human body. There is a system of nerves underneath the skin's surface known as Tactile system that send information light touch, pain, temperature, and pressure from the environment to the brain. Dysfunction within the tactile system causes misperception of sensory stimuli like touch and / or pain. This might cause self-imposed isolation, general irritability, distractibility and hyperactivity. Such children may avoid touching certain surfaces and prefers to touch specific fabrics. They sometimes avoid being touched on the face, hair or head and typically don't react to pain [8].

\section{Vestibular System Dysfunction}

The vestibular system includes structures within the internal ear (semi-circular canals) that primarily detect changes in the head position and head movement. Vestibular System Dysfunction may be hyper or hyposensitive to stimuli. Those children who are hypersensitive to vestibular stimulation present with fear and aversion to ordinary movement activities. However those who are hypersensitive may actively seek intense sensory experiences like excessive body whirling, jumping or spinning. Typically children with Vestibular dysfunction are thrill seekers. They feel seasick, when riding in car, boat, train, airplane, escalator or elevator.

\section{Proprioceptive System Dysfunction}

The proprioceptive system is associated with providing a person with a subconscious awareness of body position. The system consists of muscles, joints, and tendons. Proprioceptive system is responsible for manipulating objects during fine motor movements, like writing with a pencil and buttoning one's shirt. Children with ASD might hold the pencil tightly or might drop objects frequently. They may frequently pull, twist, or chew on things. They may bump or crash into objects. They walk along touching walls and apply much pressure when writing.

\section{Visual Input Dysfunction}

SPD in autistic children causes mismatch between the degree and intensity of the sensory input and output don't match. Based on the type of senses 
Rabindran et al., Sch J App Med Sci, September, 2020; 8(9): 2085-2089

involved there are various mismatch symptoms. Children with visual input dysfunction sometimes stare at spinning objects and whirl around their own bodies. They lack proper eye contact. They typically err in spatial relationships and bumps into individuals or things [9].

\section{Auditory Input Dysfunction}

Children with Auditory Input Dysfunction have either hyper or hypo responsiveness to sound stimulus. They have the habit of covering ears when exposed to loud sound. On the other extreme, hyporesponsive children speak louder than rest of the children [10].

\section{Taste Input Dysfunction}

Children with Taste Input Dysfunction have either hyper or hypo responsiveness to taste stimulus. They may avoid foods of certain texture or taste or eats extreme tasting foods [11]. They lick or taste playdough or toys [12].

\section{Smell Input Dysfunction}

Children with Smell Input Dysfunction have either hyper or hypo responsiveness to smell stimulus. They may feel excess smell or lack the sense of smell. They may smell everything they touch to become orientated and comfortable with the object. Typically children with this condition breathe through their mouth rather than their nose.

\section{Diagnostic Tools for SPD}

There are plenty of assessment tools for SPD diagnosis. The prominent tools include Sensory Profile 2 (SP2) [13], Sensory Integration and Praxis Test (SIPT), Sensory Processing Measure (SPM) and Evaluation in Ayres Sensory Integration (EASI) [14].

\begin{tabular}{|l|l|l|l|l|}
\hline & SP2 & SIPT & SPM & EASI \\
\hline Age Range & Birth to 14 years 11 months & 4 to 8 years 11 months & 5-12 years & 3-12 years \\
\hline Completion Time & 5-20 min & 2 hours & 15-20 min & 1-1.5 hours \\
\hline Administration & Parents & Child & Parents & Child \\
\hline
\end{tabular}

\section{Short Sensory Profile (SSP)}

Short Sensory Profile (SSP) is an abridged version of the Sensory Profile. It is a parent-report used to measure sensory behaviours. It is considered as a standardized assessment tool to evaluate sensory processing in everyday activities. It has seven subscales that assesses 1) Auditory filtering, 2) Low energy, 3) Under-responsiveness / Seeking sensation, 4) Sensitivity to movement, 5) Tactile sensation, 6) Taste / Smell and 7) Visual / Auditory [15].

\section{Test of Everyday Attention for Children (Tea-Ch)}

The TEA-CH is a standardized tool for assessing SPD in children between 6-16 years of age. It provides raw and age-corrected standard scores for each of its nine subtests, namely, 1) Sky Search, 2) Score, 3) Creature Counting, 4) Map Mission, 5) Score DT, 6) Sky Search DT, 7). Opposite Worlds, 8) Walk Don't Walk, and 9) Code Transmission [16]. Other diagnostic tools for SPD include Teacher questionnaire, Indirect classroom observations, Interval video recording, Engagement check, Questions about behavioural function (QABF), Functional assessment screening tool - revised (FAST-R), Salivary cortisol levels, Canadian occupational performance measure (COPM), Goal attainment scale (GAS), Parenting stress index- short form (PSI-SF) and Parenting sense of competence scale (PSOC), Revised Functional Behavior Assessment for Children with Sensory Integrative Dysfunction, Social Responsiveness Scale (SRS), Quick Neurological Screening Test (QNST), Vineland Adaptive Behavioural Scales, $2^{\text {nd }}$ Edition (VABS-2), Sensory experiences questionnaire (SEQ), Pervasive developmental disorder behavioural inventory (PDDBI), Autism diagnostic observation interview
(ADOS), Mail questionnaire, Caregiver interview and short observation intervals and video recording[17].

\section{Candidate Biomarkers}

Children with ASD who have SPD symptoms typically don't have neurological injury or acquired brain damage. Hence they are not picked up in diagnostic imaging tests. However Children with severe SPD have a lower basal activity of the parasympathetic nervous system compared to children with normal development. This may be a candidate for severe SPD biomarker. Moreover children with SPD have unique patterns of brain processing in EEG study that are different from those with normal development. Children with SPD show altered white matter microstructure organization in posterior cerebral tracts which connect the sensory inputs to the cognitive processing [18]. This altered microstructure can be quantified by diffusion tensor imaging (DTI). Recently plasma 8-isoprostane and cysteinyl leukotrienes have been found to be helpful as a biomarker for the early recognition of autistic patients with SPD.

\section{CONCLUSION}

Diagnosing SPD in children with ASD Spectrum Disorder is not a brief process. There is no single medical test which will diagnose it definitively; instead, in order to accurately pinpoint the child's problem, multiple evaluations and tests may be necessary. Awareness regarding the Sensory dysfunction in children with ASD will help to understand the disorder better which would ultimately lead to proper management. 


\section{REFERENCE}

1. American Psychiatric Association. Diagnostic and statistical manual of mental disorders (DSM-5®). American Psychiatric Pub; 2013 May 22.

2. Bundy A. Sensory integration: A. Jean Ayres' theory revisited. Sensory integration: Theory and practice. 2002.

3. Miller LJ, Anzalone ME, Lane SJ, Cermak SA, Osten ET. Concept evolution in sensory integration: A proposed nosology for diagnosis. American Journal of occupational therapy. 2007 Mar 1;61(2):135-40.

4. Reynolds S, Lane SJ. Diagnostic validity of sensory over-responsivity: A review of the literature and case reports. Journal of autism and developmental disorders. 2008 Mar 1;38(3):516-29.

5. Schaaf RC, Lane AE. Toward a best-practice protocol for assessment of sensory features in ASD. Journal of Autism and Developmental Disorders. 2015 May 1;45(5):1380-95.

6. Baranek GT, Foster LG, Berkson G. Tactile defensiveness and stereotyped behaviors. American Journal of Occupational Therapy. 1997 Feb 1;51(2):91-5.

7. Ashburner J, Ziviani J, Rodger S. Sensory processing and classroom emotional, behavioral, and educational outcomes in children with autism spectrum disorder. American journal of occupational therapy. 2008 Sep 1;62(5):564-73.

8. Dunn W. The sensations of everyday life: Empirical, theoretical, and pragmatic considerations. American Journal of Occupational Therapy. 2001 Nov 1;55(6):608-20.

9. Leekam S, Baron- Cohen S, Perrett D, Milders M, Brown S. Eye- direction detection: a dissociation between geometric and joint attention skills in autism. British journal of developmental psychology. 1997 Mar;15(1):77-95.
10. O'connor K. Auditory processing in autism spectrum disorder: a review. Neuroscience \& Biobehavioral Reviews. 2012 Feb 1;36(2):836-54.

11. Cermak SA, Curtin C, Bandini LG. Food selectivity and sensory sensitivity in children with autism spectrum disorders. Journal of the American Dietetic Association. 2010 Feb 1;110(2):238-46.

12. Tavassoli T, Baron-Cohen S. Taste identification in adults with autism spectrum conditions. Journal of autism and developmental disorders. 2012 Jul $1 ; 42(7): 1419-24$.

13. Chien CW, Rodger S, Copley J, Branjerdporn G, Taggart C. Sensory processing and its relationship with children's daily life participation. Physical \& occupational therapy in pediatrics. 2016 Jan 2;36(1):73-87.

14. Jorquera-Cabrera S, Romero-Ayuso D, RodriguezGil G, Triviño-Juárez JM. Assessment of sensory processing characteristics in children between 3 and 11 years old: A systematic review. Frontiers in pediatrics. 2017 Mar 30;5:57.

15. Rogers SJ, Hepburn S, Wehner E. Parent reports of sensory symptoms in toddlers with autism and those with other developmental disorders. Journal of autism and developmental disorders. 2003 Dec 1;33(6):631-42.

16. Petersen SE, Posner MI. The attention system of the human brain: 20 years after. Annual review of neuroscience. 2012 Jul 21;35:73-89.

17. Siper PM, Kolevzon A, Wang AT, Buxbaum JD, Tavassoli T. A clinician- administered observation and corresponding caregiver interview capturing DSM- 5 sensory reactivity symptoms in children with ASD. Autism Research. 2017 Jun;10(6):113340.

18. Owen JP, Marco EJ, Desai S, Fourie E, Harris J, Hill SS, Arnett AB, Mukherjee P. Abnormal white matter microstructure in children with sensory processing disorders. Neuroimage: clinical. 2013 Jan 1;2:844-53. 